Pacific Journal of Mathematics

AN INTERESTING COMBINATORIAL METHOD IN THE
THEORY OF LOCALLY FINITE SEMIGROUPS 


\title{
AN INTERESTING COMBINATORIAL METHOD IN THE THEORY OF LOCALLY FINITE SEMIGROUPS
}

\author{
T. C. BRown
}

Let $X$ be a finite set, $X^{*}$ the free semigroup (without identity) on $X$, let $M$ be a finite semigroup, and let $\varphi$ be an epimorphism of $X^{*}$ upon $M$. We give a simple proof of a combinatorial property of the triple $(X, \varphi, M)$, and exploit this property to get very simple proofs for these two theorems: 1. If $\varphi$ is an epimorphism of the semigroup $S$ upon the locally finite semigroup $T$ such that $\varphi^{-1}(e)$ is a locally finite subsemigroup of $S$ for each idempotent element $e$ of $T$, then $S$ is locally finite. 2 . Throughout 1 , replace "locally finite" by "locally nilpotent".

The method is simple enough, and yet powerful enough, to suggest its applicability in other contexts.

1. Theorem 1 below was first proved by the author in [1] by a circuitous and laborious method. In the present paper it drops out easily from Lemma 2 below, as does Theorem 2, which is new. Lemma 2 was first discovered by J. Justin ([3]) as a generalization of Lemma 1 , which is the author's ([2]). The proof given here, however, is new, and is conceptually quite transparent, though apparently nontrivial. Justin has used Lemma 2 in an alternative proof of his generalization of Van der Waerden's Theorem (on Arithmetic Progressions), using Van der Waerden's Theorem in the course of the proof. The author is inclined to believe that a refined or more powerful version of Lemma 2 would yield a proof of Van der Waerden's Theorem itself.

The construction of a sequence "in the regular way", given below, has been formalized by $R$. Rado in [4].

2. Notation and definitions. The symbol $X$ will always denote a finite set, and $X^{*}$ denotes the free semigroup without identity on $X$. Thus $X^{*}$ is the semigroup of nonempty "words" in the "letters" of the "alphabet" $X$, with juxtaposition as multiplication. If $w=$ $x_{1} x_{2} \cdots x_{k} \in X^{*}$, where the $x_{i} \in X$, then the length of $w$, denoted by $|w|$, is $k$. The symbol $X^{\omega}$ denotes the set of sequences on $X$, regarded as "infinitely long words" in the alphabet $X$. If $x, y, z \in X^{*}$ and $s \in X^{\omega}$, then $x, y$, and $z$ are each factors ( $x$ is a left factor) of the word $x y z$ and of the sequence xyzs.

Let $H$ be an infinite subset of $X^{*}$. We indicate now how to construct a sequence $s=a_{1} a_{2} \cdots \in X^{\omega}$ such that each left factor of $s$ 
is a left factor of infinitely many of the words of $H$. Such a sequence is used several times in the proofs that follow, and is said to be constructed in the regular way from $H$.

We choose the $a_{i}$ 's inductively. In view of the fact that $H$ is infinite and $X$ is finite, we choose $a_{1}$ to be an element of $X$ which occurs infinitely often as the first letter in the words of $H$, and we denote by $H_{1}$ the (infinite) set of those words of $H$ which have $a_{1}$ as first letter. Thus $a_{1}$ is a left factor of infinitely many of the words of $H$. Now suppose that $a_{1}, \cdots, a_{n} \in X$ have been chosen so that $a_{1} a_{2} \cdots a_{n}$ is a left factor of each word in an infinite subset $H_{n}$ of $H$. We choose $a_{n+1}$ to be an element of $X$ which occurs infinitely often as the $(n+1)$ st letter in the words of $H_{n}$, and denote by $H_{n \rightarrow 1}$ the (infinite) set of those words of $H_{n}$ which have $a_{n+1}$ as $(n+1)$ st letter. Thus $a_{1} a_{2} \cdots a_{n+1}$ is a left factor of infinitely many of the words of $H$.

\section{Two lemmas.}

Lemma 1. Let $s=a_{1} a_{2} \cdots \in X^{\omega}$. Then there exist an element $x \in X$ and a fixed integer $\bar{k}$ such that for any $n$ there are integers $i_{1}<i_{2}<\cdots<i_{n}$ (these depend on $n$ ) with $x=a_{i_{1}}=a_{i_{2}}=\cdots=a_{i_{n}}$ and $i_{j+1}-i_{j} \leqq \bar{k}, 1 \leqq j \leqq n-1$.

Proof. We proceed by induction on $|X|$, the cardinal of $X$. If $|X|=1$, we are through. Assume the result for $|X|=k$, and suppose now that $|X|=k+1, X=\left\{x_{1}, \cdots, x_{k+1}\right\}$. Let $s=a_{1} a_{2} \cdots \in X^{\omega}$. If $x_{k+1}$ is not missing from arbitrarily long factors of $s$ we are done, hence we may assume that there is an infinite set $H$ of factors of $s$ from which $x_{k+1}$ is missing. Thus $H \subset\left\{x_{1}, \cdots, x_{k}\right\}^{*}$, and we construct a sequence $t=b_{1} b_{2} \cdots \in\left\{x_{1}, \cdots, x_{k}\right\}^{\omega}$ from $H$ in the regular way. By the induction hypothesis, there exist an element $x \in\left\{x_{1}, \cdots, x_{k}\right\}$ and an integer $\bar{k}$ such that for any $n$ there are integers $i_{1}<i_{2}<\cdots<i_{n}$ with $x=b_{i_{1}}=b_{i_{2}}=\cdots=b_{i_{n}}$ and $i_{j+1}-i_{j} \leqq \bar{k}, 1 \leqq j \leqq n-1$. But every left factor of $t$ is a left factor of words in $H$, and each word in $H$ is a factor of $s$, therefore every left factor of $t$ is a factor of $s$, and hence every factor of $t$ is a factor of $s$, in particular the factor $b_{i_{1}} \cdots b_{i_{2}} \cdots \cdots b_{i_{n}}$. By a translation of indices (perhaps a different translation for each $n$ ) we are done.

LEMma 2. Let $\phi$ be an epimorphism of $X^{*}$ upon a finite semigroup $M$, and let $s \in X^{\omega}$. Then there exist an idempotent $e \in M$ and a fixed integer $\vec{k}$ such that for any $n$ there are $n$ consecutive factors $g_{1}, \cdots, g_{n}$ (these depend on $n$ ) of $s$ (i.e., $s=a g_{1} g_{2} \cdots g_{n} s^{\prime}$, where $\left.a, g_{1}, \cdots, g_{n} \in X^{*}, s^{\prime} \in X^{\omega}\right)$ with $e=\varphi\left(g_{1}\right)=\varphi\left(g_{2}\right)=\cdots=\varphi\left(g_{n}\right)$ and $\left|g_{j}\right| \leqq \bar{k}, 1 \leqq j \leqq n$. 
Proof. We proceed by induction on $|M|$. If $|M|=1$, we are done, so now let $M$ be fixed with $|M| \geqq 2$ and assume the result for all semigroups with cardinal smaller than that of $M$. Now with this $M$ we proceed by induction on $|X|$. If $|X|=1$ we are through, so we may assume the result for $|X|=k$ and now let $|X|=k+1$.

Let $s=a_{1} a_{2} \cdots \in X^{\omega}$, and let $x$ be a fixed element of $X$. If $x$ is missing from arbitrarily long factors of $s$, then constructing in the regular way a new sequence from the set of these factors, and arguing as in the proof of Lemma 1, we are done by the induction hypothesis on $|X|$. Thus we may assume that there is an integer $\bar{m}$ and integers $i_{1}, i_{2}, \cdots$ such that $x=a_{i_{1}}=a_{i_{2}}=\cdots$ and $0<i_{j+1}-i_{j} \leqq \bar{m}$ for $j=1,2, \cdots$. To simplify the notation, let us assume without loss of generality that $i_{1}=1$.

Next, we take a new (finite) alphabet $B=\left\{a_{i_{j}} a_{i_{j}+1} \cdots a_{i_{j+1}-1} \mid j=\right.$ $1,2, \cdots\}$ and write $s$ as a sequence in $B^{\omega}$. Let $\varphi(x)=p$. Then $\varphi\left(a_{i_{j}}\right)=p$ for $j=1,2, \cdots$, so the restriction of $\varphi$ to $B^{*}$ is an epimorphism of $B^{*}$ upon the semigroup $p M$. If $|p M|<|M|$, then we are done by the induction hypothesis on $|M|$, since we can easily find our way back to $s$ regarded as a sequence in $X^{\omega}$. (Indeed, if the length of a factor $g$ of $s$ in the alphabet $B$ is $\leqq \bar{k}$, then the length of $g$ in the alphabet $X$ is $\leqq \bar{m} \bar{k}$.)

Thus we may assume that $|p M|=|M|$, and similarly that $|M p|=$ $|M|$, for all $p \in M$ (since the fixed element $x$ chosen above was an arbitrary element of $X$ ). Since $M$ is finite, this amounts to saying that $M$ is a (finite) group.

All we have to do now is to regard $M$ temporarily as a set only, and apply Lemma 1 to the sequence $p_{1} p_{2} \cdots \varepsilon M^{\omega}$, where $p_{i}=\varphi\left(a_{1} a_{2} \cdots a_{i}\right)$, $i=1,2, \cdots$. Thus there exist an element $p \in M$ and a fixed integer $\vec{k}$ such that for any $n$ there are integrs $i_{1}<i_{2}<\cdots<i_{n}$ with $p=p_{i_{1}}=$ $p_{i_{2}}=\cdots p_{i_{n}}$ and $i_{j+1}-i_{j} \leqq \bar{k}, 1 \leqq j \leqq n-1$. Setting $g_{1}=a_{1} \cdots a_{i_{1}}, g_{2}=$ $a_{i_{1}+1} \cdots a_{i_{2}}, \cdots, g_{n}=a_{i_{n-1}+1} \cdots a_{i_{n}}$, this says $\varphi\left(g_{1}\right)=\varphi\left(g_{1} g_{2}\right)=\cdots=$ $\varphi\left(g_{1} g_{2} \cdots g_{n}\right)$, an so $e=\varphi\left(g_{2}\right)=\varphi\left(g_{3}\right)=\cdots=\varphi\left(g_{n}\right)$ (where $e$ is the identity of the group $M$ ), which is the conclusion we seek.

\section{Two theorems.}

THEOREM 1. Let $\phi$ be an epimorphism of the semigroup $S$ upon the locally finite semigroup $T$ such that $\varphi^{-1}(e)$ is a locally finite subsemigroup of $S$ for each idempotent element $e$ of $T$. Then $S$ is locally finite.

Proof. First we note that if suffices to consider the case where $T$ is finite. (For suppose the theorem is true in this case, and let $\varphi$ be 
an epimorphism (with the required properties) of $S$ onto an arbitrary, that is, possibly infinite, locally finite semigroup $T^{\prime}$. Let $X$ be a finite subset of $S$, and let $\langle X\rangle$ denote the subsemigroup of $S$ generated by $X$. It is required to show that $\langle X\rangle$ is finite. Now $\langle\varphi(X)\rangle T$ is a finite subsemigroup of $T^{\prime}$ since $T^{\prime}$ is locally finite, hence restricting $\varphi$ to $\varphi^{-1}(T)$ we get an epimorphism (with the required properties) of $\varphi^{-1}(T)$ onto the finite semigroup $T$. By our assumption, $\phi^{-1}(T)$ is locally finite, hence, since $X \subset \varphi^{-1}(T),\langle X\rangle$ is finite, as required.)

Therefore we assume that $T$ is finite, and we let $X$ denote a finite subset of $S,\langle X\rangle$ the subsemigroup of $S$ generated by $X$.

To show that $\langle X\rangle$ is finite, it is convenient to introduce some additional notation. Let $X=\left\{x_{1}, \cdots, x_{m}\right\}$, and let $\bar{X}=\left\{\bar{x}_{1}, \cdots, \bar{x}_{m}\right\}$ be a set. If $\bar{w}=\bar{x}_{i_{1}} \cdots \bar{x}_{i_{k}} \in \bar{X}^{*}$, let $w$ denote the element $x_{i_{1}} \cdots x_{i_{k}}$ of $\langle X\rangle$. Thus "removal of bars" is a homomorphism of $\bar{X}^{*}$ upon $\langle X\rangle$. We shall call a word $\bar{w} \in \bar{X}^{*}$ contractible if there is another word $\bar{u} \in \bar{X}^{*}$ such that $|\bar{u}|<|w|$ and $u=w$. A sequence $\bar{s} \in \bar{X}^{\omega}$ is contractible if $\bar{s}$ has a contractible factor. Now $\langle X\rangle$ will be finite provided every sufficiently long word of $\bar{X}^{*}$ is contractible, and this will be the case provided that every sequence in $\bar{X}^{\omega}$ is contractible; for otherwise we could take an infinite set of noncontractible words of $\bar{X}^{*}$ and, by then constructing a sequence in the regular way from this set, obtain a non-contractible sequence.

Thus it remains to show that every sequence in $\bar{X}^{\omega}$ is contractible.

Let $\bar{s} \in \bar{X}^{\omega}$, and define the homomorphism $\bar{\varphi}$ from $\bar{X}^{*}$ into $T$ by setting $\bar{\varphi}(\bar{w})=\varphi(w)$ for $\bar{w} \in \bar{X}^{*}$. Applying Lemma 2, we obtain an idempotent $e \in T$ and a fixed integer $\bar{k}$ such that for any $n$ there are $n$ consecutive factors $\bar{g}_{1}, \cdots, \bar{g}_{n}$ of $\bar{s}$ with $e=\bar{\varphi}\left(\bar{g}_{1}\right)=\cdots=\bar{\phi}(\bar{g})$ and $\left|\bar{g}_{j}\right| \leqq \bar{k}, 1 \leqq j \leqq n$. By the definition of $\bar{\varphi}$, we have $g_{1}, \cdots, g_{n}$ all in $\varphi^{-1}(e)$, which is locally finite by assumption. Since $\left|\bar{g}_{j} \leqq \bar{k}\right|$ there are only finitely many possibilities for the elements $g_{1}, \cdots, g_{n}$, hence the element $g_{1} \cdots g_{n}$ always belongs to a certain fixed finite subsemigroup of $\varphi^{-1}(e)$, no matter how large $n$ is. Thus if $n$ is taken sufficiently large, the factor $\bar{g}_{1} \cdots \bar{g}_{n}$ of $\bar{s}$ will be contractible. Thus the sequence $\bar{s}$ is contractible. This completes the proof.

THEOREM 2. Let $\varphi$ be an epimorphism of the semigroup $S$ upon the locally nilpotent semigroup $T$ such that $\varphi^{-1}(e)$ is a locally nilpotent subsemigroup of $S$ for each idempotent element e of $T$. Then $S$ is losally nilpotent.

Proof. The proof of Theorem 2 is practically the same as the proof of Theorem 1. Here instead of showing that every sequence $\bar{s}$ in $\bar{X}^{\omega}$ (same notation as in the proof of Theorem 1) has a contractible factor, one shows that every sequence $\bar{s}$ has a factor $w$ with $w=0$. 


\section{REFERENCES}

1. T. C. Brown, On locally finite semigroups, (in Russian) Ukrainian Math. J., 20 No. 6 (1968), 732-738.

2. - Van der Waerden's theorem on arithmetic progressions, Amer. Math. Soc., Notices 16 No. 1 (1969), 245.

3. J. Justin, Généralisation du théorème de Van der Waerden sur les semi-groups répétitifs, to appear in J. Combinatorial Theory.

4. R. Rado, Axiomatic treatment of rank in infinite sets, Canadian J. Math., 1 (1949), 337-343.

Received November 3, 1969. Partially supported by Canadian N.R.C. Grant No. A-3982.

Simon Fraser University 



\section{PACIFIC JOURNAL OF MATHEMATICS}

\section{EDITORS}

H. SAMELson

Stanford University

Stanford, California 94305

R. R. PHelPS

University of Washington

Seattle, Washington 98105
J. Dugundu

Department of Mathematics

University of Southern Californle

Los Angeles, California 9.0007

RICHARD ARENS

University of California

Los Angeles, California 9.0024

\section{ASSOCIATE EDITORS}
E. F. BECKENBACH
B. H. NeumanN
F. WOLE
K. Yoshida

\section{SUPPORTING INSTITUTIONS}

UNIVERSITY OF BRITISH COLUMBIA

CALIFORNIA INSTITUTE OF TECHNOLOGY

UNIVERSITY OF CALIFORNIA

MONTANA STATE UNIVERSITY

UNIVERSITY OF NEVADA

NEW MEXICO STATE UNIVERSITY

OREGON STATE UNIVERSITY

UNIVERSITY OF OREGON

OSAKA UNIVERSITY

UNIVERSITY OF SOUTHERN CALIFORNIA

\author{
STANFORD UNIVERSITY \\ UNIVERSITY OF TOKYO \\ UNIVERSITY OF UTAH \\ WASHINGTON STATE UNIVERSITY \\ UNIVERSITY OF WASHINGTON \\ AMERICAN MATHEMATICAL SOCIETY \\ CHEVRON RESEARCH CORPORATION \\ NAVAL WEAPONS CENTER
}

The Supporting Institutions listed above contribute to the cost of publication of this Journal, but they are not owners or publishers and have no responsibility for its content or policies.

Mathematical papers intended for publication in the Pacific Journal of Mathematics should be in typed form or offset-reproduced, (not dittoed), double spaced with large margins. Underline Greek letters in red, German in green, and script in blue. The first paragraph or two must be capable of being used separately as a synopsis of the entire paper. The editorial "we" must not be used in the synopsis, and items of the bibliography should not be cited there unless absolutely necessary, in which case they must be identified by author and Journal, rather than by item number. Manuscripts, in duplicate if possible, may be sent to any one of the four editors. Please classify according to the scheme of Math. Rev. Index to Vol. 39. All other communications to the editors should be addressed to the managing editor, Richard Arens, University of California, Los Angeles, California, 90024.

50 reprints are provided free for each article; additional copies may be obtained at cost in multiples of 50 .

The Pacific Journal of Mathematics is published monthly. Effective with Volume 16 the price per volume (3 numbers) is $\$ 8.00$; single issues, $\$ 3.00$. Special price for current issues to individual faculty members of supporting institutions and to individual members of the American Mathematical Society: $\$ 4.00$ per volume; single issues $\$ 1.50$. Back numbers are available.

Subscriptions, orders for back numbers, and changes of address should be sent to Pacific Journal of Mathematics, 103 Highland Boulevard, Berkeley, California, 94708.

PUBLISHED BY PACIFIC JOURNAL OF MATHEMATICS, A NON-PROFIT CORPORATION

Printed at Kokusai Bunken Insatsusha (International Academic Printing Co., Ltd.), 7-17, Fujimi 2-chome, Chiyoda-ku, Tokyo, Japan. 


\section{Pacific Journal of Mathematics}

\section{Vol. 36, No. 2 December, 1971}

George E. Andrews, On a partition problem of H. L. Alder ............ 279

Thomas Craig Brown, An interesting combinatorial method in the theory of locally finite semigroups .......................... 285

Yuen-Kwok Chan, A constructive proof of Sard's theorem ............. 291

Charles Vernon Coffman, Spectral theory of monotone Hammerstein

operators...................................... 303

Edward Dewey Davis, Regular sequences and minimal bases .......... 323

Israel (Yitzchak) Nathan Herstein and Lance W. Small, Regular elements in

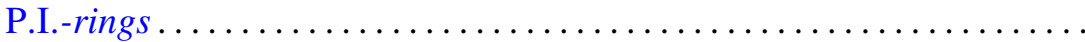

Marcel Herzog, Intersections of nilpotent Hall subgroups ..............

W. N. Hudson, Volterra transformations of the Wiener measure on the space

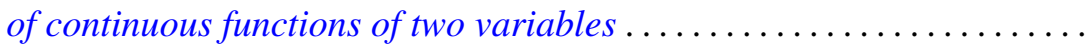

J. H. V. Hunt, An n-arc theorem for Peano spaces ................ 351

Arnold Joseph Insel, A decomposition theorem for topological group

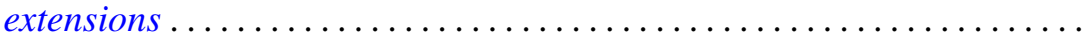

Caulton Lee Irwin, Inverting operators for singular boundary value

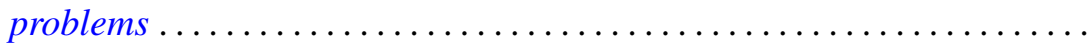

Abraham A. Klein, Matrix rings of finite degree of nilpotency ............ 387

Wei-Eihn Kuan, On the hyperplane section through a rational point of an algebraic variety...

John Hathway Lindsey, II, On a six-dimensional projective representation of $\mathrm{PSU}_{4}(3)$

Jorge Martinez, Approximation by archimedean lattice cones ...

J. F. McClendon, On stable fiber space obstructions .........

Mitsuru Nakai and Leo Sario, Behavior of Green lines at the Kuramochi boundary of a Riemann surface ....................

Donald Steven Passman, Linear identities in group rings. I. .

Donald Steven Passman, Linear identities in group rings. II ...

David S. Promislow, The Kakutani theorem for tensor products of

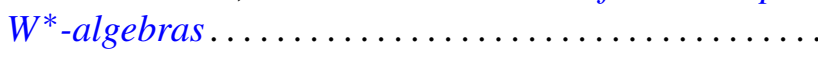

Richard Lewis Roth, On the conjugating representation of a finite group

Bert Alan Taylor, On weighted polynomial approximation of entire functions...

William Charles Waterhouse, Divisor classes in pseudo Galois

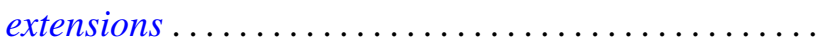

Chi Song Wong, Subadditive functions ...

Ta-Sun $\mathrm{Wu}$, A note on the minimality of certain bitransformation groups 